\title{
Inaugural Chinese Planetary Science Conference
}

\author{
Held in Suzhou, Jiangsu province of China in June 2021, the conference served to unite a wide community of \\ planetary science within China, and hopes to become one of the world's premier planetary science conferences in \\ the future.
}

A bout twenty years ago, China did not have its own spacecraft mission to study space physics exclusively, let alone any missions to the Moon and to Mars. Who could have imagined that China would successfully implement the Double Star mission ${ }^{1}$ to explore Earth's magnetosphere, the series of lunar missions Chang'E-1, -2, $-3,-4,-5^{2}$, and the first Mars mission Tianwen- $1^{3,4}$ in the space of two decades? These successful missions have undoubtedly driven the rapid development of China's planetary science community. This is because more and more planetary scientists are required for analysing the scientific data returned by these spacecraft, and more scientific requirements are presented by scientists to guide the scientific goals of future missions.

In such circumstances, various different branches of planetary science and their corresponding communities have developed gradually in China, for example, planetary space physics, planetary atmospheres, planetary geology, planetary geochemistry, planetary interior, exoplanets, terrestrial small bodies, astrobiology, and so on. Meanwhile, a few universities, represented by the University of Chinese Academy of Sciences (UCAS), have launched associated courses to educate students in planetary science. The academic consortia and organizations of planetary science have also been established, such as the China University Planetary Science Alliance, the Lunar Science and Comparative Planetology Committee of the Chinese Space Science Society, the Planetary Committee of the Chinese Astronomical Society, and the Planetary Physics Committee of the Chinese Geophysical Society. Moreover, to disseminate original research output in planetary science in China, the international peer-reviewed journal Earth and Planetary Physics ${ }^{5}$ was launched in 2017.

In contrast to the booming development of China's planetary science, China, however, had not yet held its own national planetary conference. Due to this situation, many Chinese scientists have previously had to attend other world-wide conferences, such as the Lunar and Planetary Science Conference (LPSC) and the Europlanet Science Congress (EPSC) to seek academic

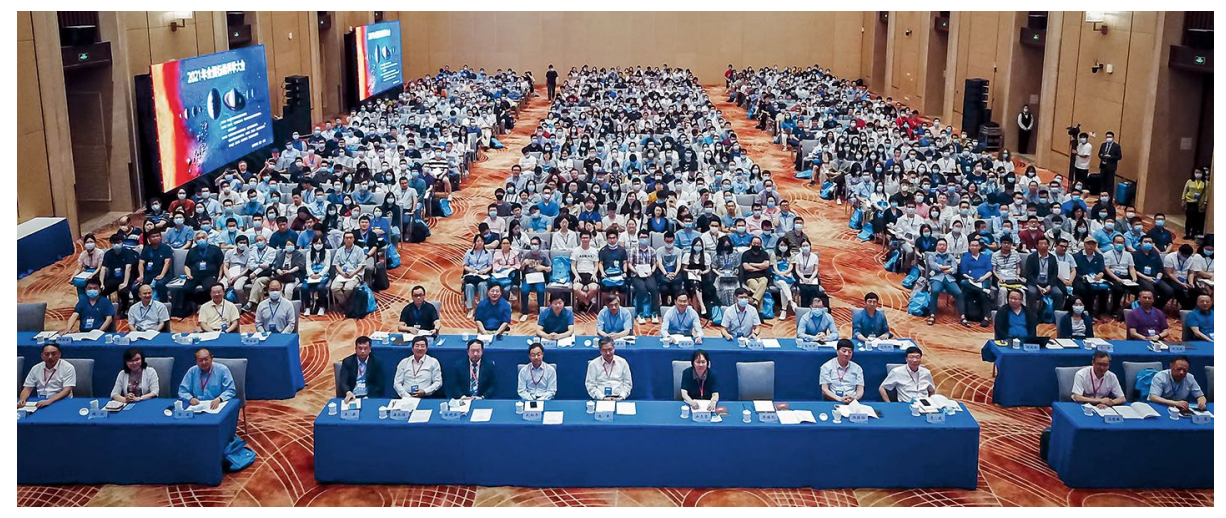

Fig. 1 | The group photo of attendees of the first Chinese Planetary Science Conference on 19 June 2021, held in Suzhou, Jiangsu province of China. 1,020 attendees from 106 institutions participated. Credit: The Planetary Physics Committee of the Chinese Geophysical Society.

communication. Given the rapid global development of planetary science and the current state of the COVID-19 pandemic overseas, Chinese scientists are more eager than ever to attend a nationally held conference. The time seemed ripe to hold a comprehensive conference for the communities of planetary science in China.

The conference, the inaugural Chinese Planetary Science Conference (CPSC), organized by the Planetary Physics Committee of the Chinese Geophysical Society, took place on 18-21 June 2021 in Suzhou, Jiangsu province of China (Fig. 1). CPSC received about 500 abstracts covering a wide range of disciplines within planetary science, including planetary space environment, planetary atmospheres, planetary geology, planetary interior dynamics, asteroids, comets, exoplanets, biology, future missions to space, and probing techniques, for example. It is also astounding to note that the conference attracted 1,020 attendees together from 106 different institutions, including the attendees from the universities of Macau and Hong Kong, which is comparable to that of the EPSC (regularly around 1,000 attendees each year), and about half that of the LPSC (regularly around 1,800 attendees each year) (Fig. 2). It is regretful that the CPSC is not open to the world yet due to COVID-19 restrictions on travel. The sheer number of attendees demonstrates that planetary science within China has developed rapidly in recent years and the number of planetary scientists has already grown to a massive scale.

Overall, some clear conclusions regarding the current status of China's planetary science can be drawn from this conference:

- Half of the attendees were students, and female scientists made up nearly a quarter of the total attendees. Thus, the current community of China's planetary scientist is very youthful, and female scientists play an important role in this community.

- About half of the attendees were from the institutes of the Chinese Academy and Sciences (CAS), which means that the main force to conduct planetary science in China is led by the CAS.

- The abstracts received by the conference covered various aspects of planetary science, which implies that planetary science is being treated as a system science, and the interdisciplinary nature of planetary science in China is maturing.

- The subjects of planetary space environment and planetary geology dominated the received abstracts, which suggests that the communities of planetary space environment and planetary geology are of particular importance to the current study of planetary science in China.

- To encourage and facilitate comprehensive discussions, most presentations, including the presentations of graduate 

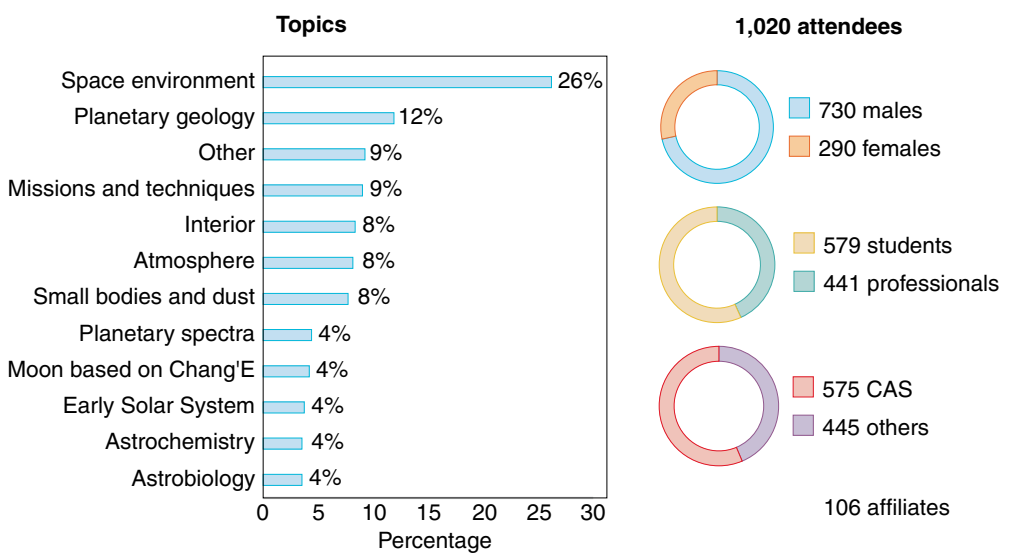

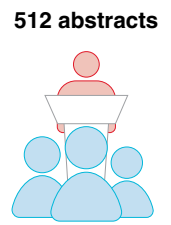

404 speakers

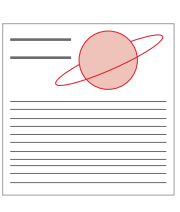

108 posters
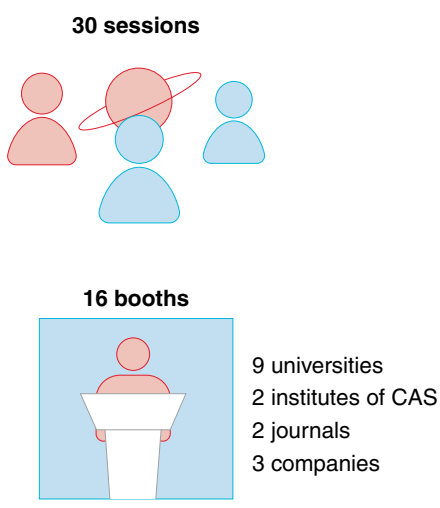

Fig. 2 | The first CPSC. An overview with respect to the numbers of attendees, abstracts, topics, sessions, and booths.

students, were delivered as 15-minute oral presentations, which strengthens the training and education of young scientists, in particular. In contrast, both EPSC and LPSC assigned oral presentations only for a minority of attendees.

Several striking takeaways from this conference demonstrated that: (1) China will launch an optical telescope with a 2-m aperture, China Space Station Telescope around 2024, to detect celestial bodies within the Solar System and cosmos ${ }^{6}$; (2) China is planning to launch a spacecraft around 2024, with the aim of flying to $100 \mathrm{au}$ and beyond until approximately 2049 , allowing it to explore the boundary of the Solar System or heliopause (3) the future of China's lunar missions will gradually shift to a study of the Moon's interior, as China will look to establish research stations on the lunar surface with international collaborations;

(4) China is actively developing its discipline of modern planetary science, and the Chinese universities, represented by UCAS, have already made significant progress in facilitating the growth of the education system around planetary science ${ }^{8-13}$.

It is highly encouraging to anticipate that, with the end of COVID-19, CPSC would be allowed to open to the whole world, thereby facilitating CPSC's ability to become one of the world's premier annual planetary science conferences in the future. Moreover, we expect that more and more international planetary scientists will participate in this conference and become a part of the global collaboration with China to explore the mysteries of planets, for the common benefit of all humankind.

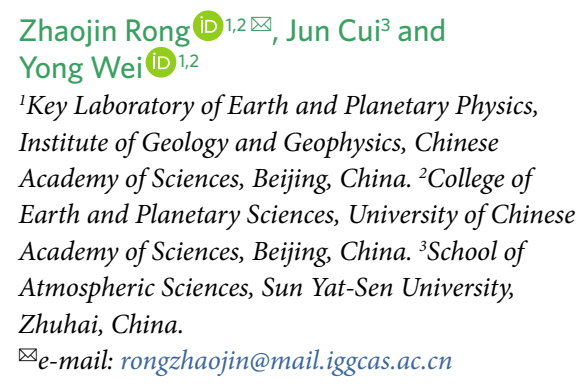

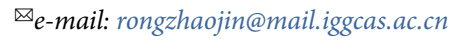

Published online: 5 October 2021

https://doi.org/10.1038/s41550-021-01503-Z

References

1. Liu, Z. X. et al. Ann. Geophys. 23, 2707-2712 (2005).

2. Li, C. L., Wang, C., Wei, Y. \& Lin, Y. T. Science 365 , 238-239 (2019)

3. Wan, W. X., Wang, C., Li, C. L. \& Wei, Y. Nat. Astron. 4, 721 (2020).

4. Normile, D. Science 368, 6498 (2020).

5. Wan, W. X. Earth Planet. Phys. http://www.eppcgs.org/ fileDQYXXWL/journal/article/dqyxxwl/2017/1/PDF/ wanweixing.pdf (2017).

6. Zhan, H. Chin. Sci. Bull. [in Chinese] 66, 1290-1298 (2021).

7. Wu, W. R. et al. Sci. Sin. Inform. [in Chinese] 49, 1-16 (2019).

8. Wu, F. Y. et al. Bull. Chin. Acad. Sci. [in Chinese] $\mathbf{3 4}$ 741-747 (2019).

9. Wan, W. X. et al. Bull. Chin. Acad. Sci. [in Chinese] 34, 748-755 (2019)

10. Wei, Y. \& Zhu, R. X. Bull. Chin. Acad. Sci. [in Chinese] 34, 756-759 (2019).

11. Rong, Z. J. et al. Bull. Chin. Acad. Sci. [in Chinese] 34, 760-768 (2019)

12. Hui, H. J. \& Qin, L. P. Bull. Chin. Acad. Sci. [in Chinese] 34, 769-775 (2019).

13. Li, X. Y. et al. Bull. Chin. Acad. Sci. [in Chinese] 34, 776-784 (2019).

Competing interests

The authors declare no competing interests. 